\title{
Osimertinib-induced interstitial lung disease after treatment with anti-PD1 antibody
}

\author{
Nobuaki Mamesaya ${ }^{1} \cdot$ Hirotsugu Kenmotsu ${ }^{1} \cdot$ Mineo Katsumata $^{2} \cdot$ Takashi Nakajima $^{3}$. \\ Masahiro Endo ${ }^{4} \cdot$ Toshiaki Takahashi $^{1}$
}

Received: 9 August 2016 / Accepted: 31 August 2016/Published online: 6 September 2016

(C) The Author(s) 2016. This article is published with open access at Springerlink.com

\begin{abstract}
Summary We report a case of a 38-year-old woman who was diagnosed with stage IV lung adenocarcinoma, harboring an epidermal growth factor receptor $(E G F R) \mathrm{L} 858 \mathrm{R}$ mutation on exon 21 and a T790 M mutation on exon 20. The patient was treated with osimertinib, a third-generation EGFR tyrosine kinase inhibitor (EGFR-TKI) following treatment with nivolumab, an anti-Programmed Cell Death 1 (anti-PD1) antibody. After initiating osimertinib treatment, the patient began to complain of low-grade fever and shortness of breath without hypoxemia, and her chest radiograph and a CT scan revealed a remarkable antitumor response, although faint infiltrations were observed in the bilateral lung field. Bronchoalveolar lavage fluid mainly contained lymphocytes (CD4+/CD8+ ratio of 0.3 ), and a transbronchial lung biopsy specimen showed lymphocytic alveolitis with partial organization in several alveolar spaces. Therefore we diagnosed the patient with osimertinib-induced interstitial lung disease (ILD) after treatment with anti-PD1 antibody. We considered anti-PD1 therapies may be the risk factor of EGFR-TKI-induced ILD.
\end{abstract}

Hirotsugu Kenmotsu

h.kenmotsu@scchr.jp

1 Division of Thoracic Oncology, Shizuoka Cancer Center Hospital, 1007 Shimonagakubo, Nagaizumi-cho, Sunto-gun,

Shizuoka 411-8777, Japan

2 Department of Pulmonary Medicine, Seirei Hamamatsu General Hospital, Shizuoka, Japan

3 Division of Pathology, Shizuoka Cancer Center Hospital, Shizuoka, Japan

4 Division of Diagnostic Radiology, Shizuoka Cancer Center Hospital, Shizuoka, Japan
Keywords Lung cancer $\cdot E G F R$ mutation $\cdot E G F R$ tyrosine kinase inhibitor $\cdot$ Anti-PD1 antibody $\cdot$ Interstitial lung disease

\section{Case report}

A 38-year-old woman with no smoking history noticed right shoulder pain and dyspnea on exertion in December 2014, and presented at hospital in January 2015. She was diagnosed with stage IV lung adenocarcinoma, harboring an epidermal growth factor receptor (EGFR) L858R mutation on exon 21. She was treated with bevacizumab plus erlotinib as first-line chemotherapy, and subsequently received carboplatin plus pemetrexed. In February 2016, nivolumab at a dose of $3 \mathrm{mg} /$ $\mathrm{kg}$ was administered every 2 weeks up to four cycles. However, no significant response was observed, and a CTguided needle biopsy of the pleural lesion was performed to evaluate EGFR mutation status. The biopsy specimens revealed adenocarcinoma harboring an EGFR L858R mutation on exon 21 and a T790 M mutation on exon 20. The patient was referred to our institution in April 2016 to be treated with osimertinib, a third-generation $E G F R$ tyrosine kinase inhibitor (EGFR-TKI). As the tumor had shown dramatic and aggressive growth since diagnosis, osimertinib treatment ( $80 \mathrm{mg}$, once daily) was initiated 8 days after the last administration of nivolumab.

Thirty-one days after initiating osimertinib treatment, the patient began to complain of low-grade fever and shortness of breath without hypoxemia. Her chest radiograph revealed a remarkable antitumor response with an improvement in right pleural thickening and pleural effusion, although faint infiltrations were observed in the bilateral lung field. Because the possibility of osimertinib-induced interstitial lung disease (ILD) was considered, treatment with osimertinib was discontinued. After osimertinib treatment interruption, the 
Fig. 1 Chest $\mathrm{CT}$ findings before and after treatment. a Solid tumors with intrapulmonary metastasis and no evidence of preexisting interstitial pneumonia before treatment with osimertinib. b Remarkable shrinkage of tumors after treatment with osimertinib. c, d Diffuse, faint, ground-glass opacities lacking consolidation and traction bronchiectasis in both lungs after treatment with osimertinib
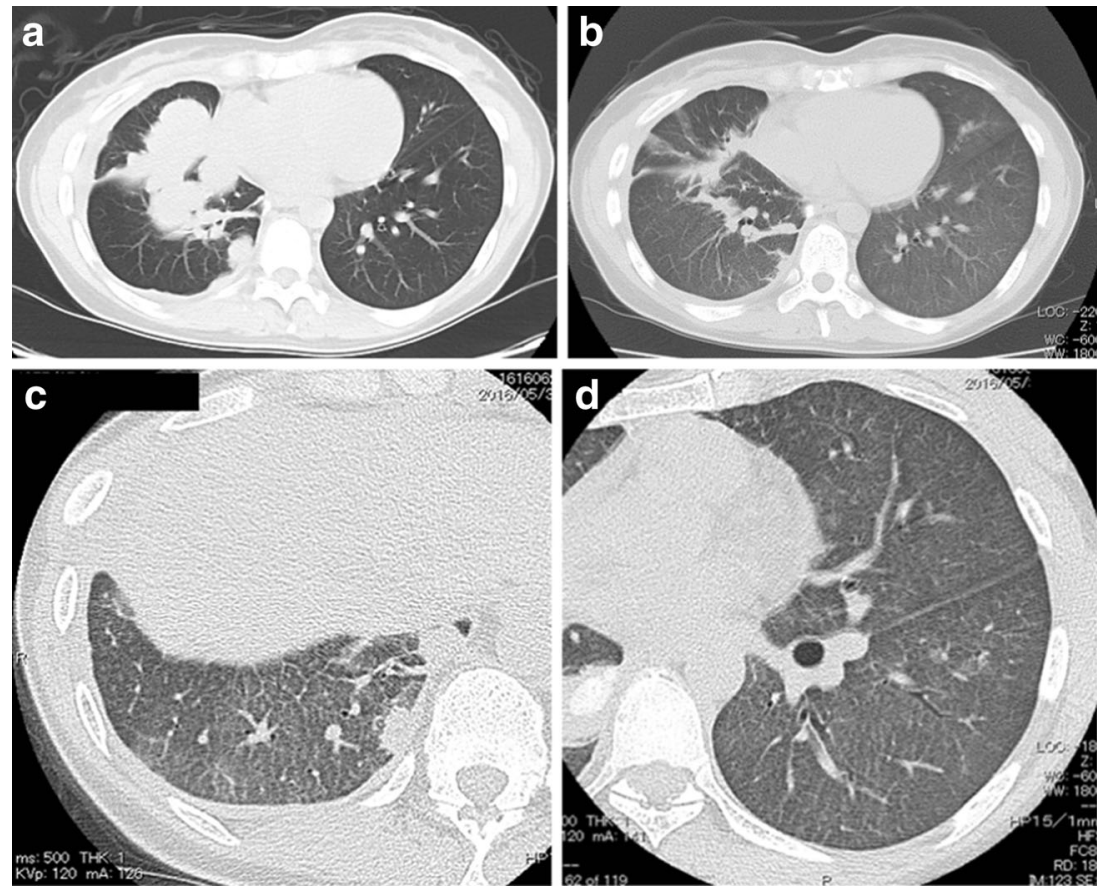

patient's symptoms were gradually relieved and osimertinib was re-administered following consideration of the clinical benefit of its antitumor efficacy. A week after resumption of osimertinib treatment, however, her symptoms worsened and bilateral faint shadows were observed by chest radiograph. Furthermore, the chest CT scan revealed further tumor shrinkage and bilateral diffuse, faint, ground-glass opacities lacking consolidation and traction bronchiectasis, a pattern compatible with hypersensitivity pneumonitis (Fig. 1). Thus, osimertinibinduced ILD was strongly suspected and we performed various appropriate examinations.

Bronchoalveolar lavage fluid (BALF), obtained from the left bronchus $(90 \mathrm{~mL} / 150 \mathrm{~mL})$, contained a white blood count of 1660 cells $/ \mu \mathrm{L}$ ( $83 \%$ lymphocytes, $14.5 \%$ macrophages, $1 \%$ neutrophils, and $1 \%$ eosinophils) and showed a CD4+/ $\mathrm{CD} 8+$ ratio of 0.3 . BALF culture was negative for pathogens including bacteria, fungi, acid-fast bacilli, and pneumocystis jirovecii by polymerase chain reaction. A transbronchial lung biopsy specimen showed mainly lymphocytic alveolitis with partial organization in several alveolar spaces, without severe fibrosis. There was no evidence of infection, cardiogenic pulmonary edema, carcinomatous lymphangitis, pulmonary hemorrhage, or hyaline membranes (Fig. 2). This pathological pattern was compatible with previous reports of druginduced ILD, although there are few reports on the specific pathological features of EGFR-TKI-induced ILD [1].
Fig. 2 Transbronchial lung biopsy specimen following treatment with osimertinib. a, b Lymphocytic alveolitis with partial organization (HE staining at low- and high-powered fields). c Strong CD8+ cytotoxic T-cell lymphocyte-positive infiltrate was mainly distributed in the alveolar septum (CD8 immunostaining at high-powered field). d Weak CD4+ helper T-cell lymphocyte-positive infiltrate mainly distributed in the alveolar septum (CD4 immunostaining at highpowered field)
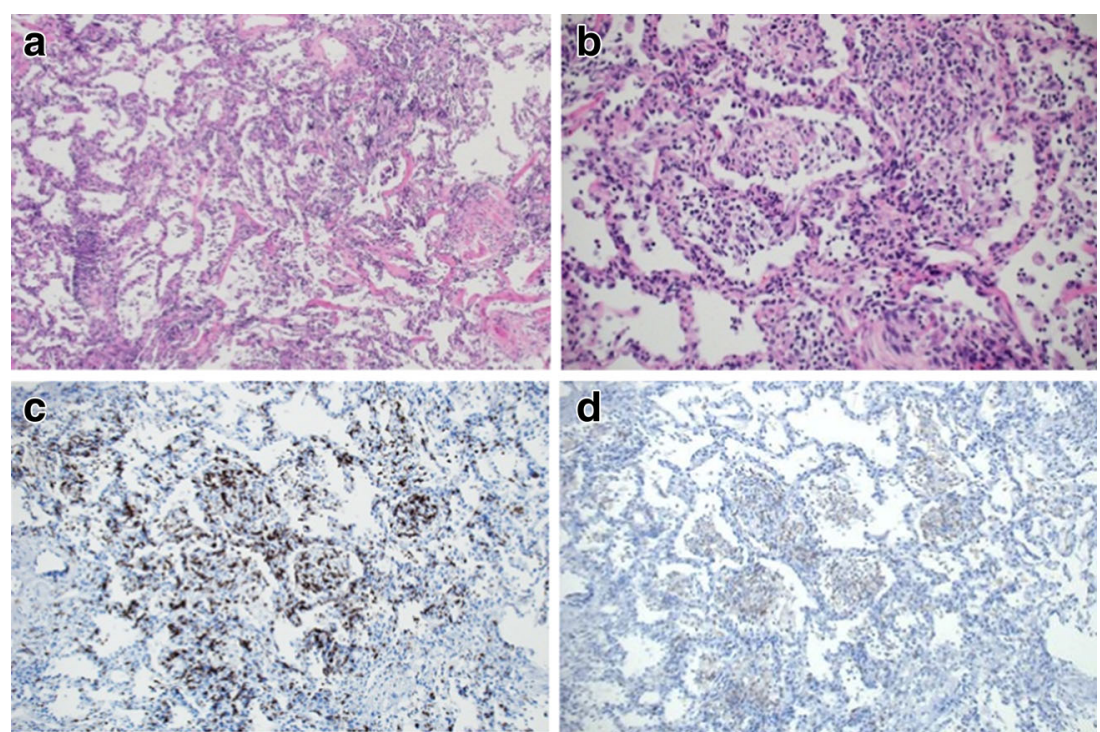
Although general blood tests including serum inflammatory markers, white blood cell count, and CRP level were within the normal range, serum KL-6 was elevated from $547 \mathrm{U} / \mathrm{mL}$ (May 2016) to $2310 \mathrm{U} / \mathrm{mL}$ (June 2016). Based on these clinical findings, we diagnosed the patient with osimertinibinduced ILD. Following withdrawal of osimertinib, her symptoms and the faint shadows on the bilateral lung field gradually diminished without administration of steroids, further supporting the diagnosis of osimertinib-induced ILD. Two months after discontinuation of osimertinib treatment, a chest CT scan showed that the bilateral diffuse, faint, ground-glass opacities had disappeared completely, but right pleural thickening had worsened. Therefore, she has been treated with ramucirumab plus docetaxel as subsequent chemotherapy from August 2016.

\section{Discussion}

To our knowledge, this is the first case report of pathologically proven lung injury caused by osimertinib. Osimertinib is effective against both $E G F R$-sensitizing and T790 M mutations, and has recently been approved in Japan. Incidence of osimertinib-induced ILD was reported as $3 \%$, and grade 5 was observed in $1 \%$ of cases [2]. However, the incidence of anti-Programmed Cell Death 1 (anti-PD1) antibody-induced pulmonary toxicities has been reported as $9 \%$, with grade 5 observed in $2 \%$ of cases [3]. Recently, updated results from the osimertinib plus durvalumab (MEDI4736) antiProgrammed Cell Death 1 Ligand 1 monoclonal antibody combination arm of the TATTON study (NCT02143466) have been reported [4]. ILD was observed in $38 \%$ of patients treated with the combination of osimertinib plus durvalumab. Anti-PD1 therapies may potentially enhance immunological reactions related to CD8+ cytotoxic T-cell lymphocyte, including osimertinib-induced ILD. In this case, nivolumab administered 1 week before treatment with osimertinib was considered to be a possible risk factor for EGFR-TKI-induced ILD.

In conclusion, we report radiological and pathological findings of osimertinib-induced ILD following treatment with an anti-PD1 antibody. The possibility of increasing the risk of $E G F R$-TKI-induced ILD after anti-PD1 antibody therapy therefore exists. Osimertinib-induced ILD has been previously reported and can be associated with fatal adverse events. Careful monitoring of the patient's clinical course for any relevant symptoms to ensure early detection of ILD may be important to prevent the development of life-threatening ILD.
Further studies are warranted, however, to clarify the risk factors for osimertinib-induced ILD.

Acknowledgments We wish to thank H. Nakamura (Department of Pulmonary Medicine, Seirei Hamamatsu General Hospital, Shizuoka, Japan), K. Nakashima, S Omori, A. Ono, T. Naito, and, H. Murakami (Division of Thoracic Oncology, Shizuoka Cancer Center Hospital, Shizuoka, Japan) for comments and helpful discussions on earlier version of this paper.

\section{Compliance with ethical standards}

Conflicts of interest $\mathrm{HK}$ has received grants and honoraria from AstraZeneca K.K., personal fees from ONO PHARMACEUTICAL CO., LTD. TT has received grants and honoraria from AstraZeneca K.K., grants and honoraria from ONO PHARMACEUTICAL CO., LTD. ME has received honoraria from ONO PHARMACEUTICAL CO., LTD. NM has no conflicts of interest to declare. MK has no conflicts of interest to declare. TN has no conflicts of interest to declare.

Funding There is no funding about this case report.

Ethical approval All procedures performed in the human participant were in accordance with the ethical standards of the institutional and/or national research committee and with the 1964 Helsinki declaration and its later amendments or comparable ethical standards.

Informed consent Informed consent was obtained from the patient.

Open Access This article is distributed under the terms of the Creative Commons Attribution 4.0 International License (http:// creativecommons.org/licenses/by/4.0/), which permits unrestricted use, distribution, and reproduction in any medium, provided you give appropriate credit to the original author(s) and the source, provide a link to the Creative Commons license, and indicate if changes were made.

\section{References}

1. Kubo K, Azuma A, Kanazawa M, et al. (2013) Consensus statement for the diagnosis and treatment of drug-induced lung injuries. Respir Investig 51(4):260-277

2. Yang J, Ramalingam SS, Jänne PA, Cantarini M, Mitsudomi T (2016) LBA2 PR Osimertinib (AZD9291) in pre-treated pts with T790 M-positive advanced NSCLC: updated Phase 1 (P1) and pooled Phase 2 (P2) results. J Thorac Oncologia 11(4 Suppl): S152-S153. doi:10.1016/S1556-0864(16)30325-2

3. Gettinger SN, Horn L, Gandhi L, et al. (2015) Overall survival and long-term safety of nivolumab (anti-programmed death 1 antibody, BMS-936558, ONO-4538) in patients with previously treated advanced non-small-cell lung cancer. J Clin Oncologia 33(18):2004-2012

4. Ahn MJ, Yang J, Yu H, et al. (2016) 136O: osimertinib combined with durvalumab in EGFR-mutant non-small cell lung cancer: results from the TATTON phase Ib trial. J Thorac Oncol 11(4 Suppl):S115. doi:10.1016/S1556-0864(16)30246-5 